\title{
Row-strict quasisymmetric Schur functions
}

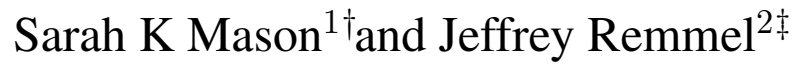 \\ ${ }^{1}$ Department of Mathematics, Wake Forest University, Winston-Salem, NC, USA \\ ${ }^{2}$ Department of Mathematics, University of California, San Diego, La Jolla, CA, USA
}

\begin{abstract}
Haglund, Luoto, Mason, and van Willigenburg introduced a basis for quasisymmetric functions called the quasisymmetric Schur function basis which are generated combinatorially through fillings of composition diagrams in much the same way as Schur functions are generated through reverse column-strict tableaux. We introduce a new basis for quasisymmetric functions called the row-strict quasisymmetric Schur function basis which are generated combinatorially through fillings of composition diagrams in much the same way as Schur functions are generated through row-strict tableaux. We describe the relationship between this new basis and other known bases for quasisymmetric functions, as well as its relationship to Schur polynomials. We obtain a refinement of the omega transform operator as a result of these relationships.

Résumé. Haglund, Luoto, Mason, et van Willigenburg ont introduit une base pour les fonctions quasi-symétriques appelée base des fonctions de Schur quasi-symétriques, qui sont construites en remplissant des diagrammes de compositions, d'une manière très semblable à la construction des fonctions de Schur à partir des tableaux "column-strict" (ordre strict sur les colonnes). Nous introduisons une nouvelle base pour les fonctions quasi-symétriques appelée base des fonctions de Schur quasi-symétriques "rowstrict”, qui sont construites en remplissant des diagrammes de compositions, d'une manière très semblable à la construction des fonctions de Schur à partir des tableaux "row-strict" (ordre strict sur les lignes). Nous décrivons la relation entre cette nouvelle base et d'autres bases connues pour les fonctions quasi-symétriques, ainsi que ses relations avec les polynômes de Schur. Nous obtenons un raffinement de l'opérateur oméga comme conséquence de ces relations.
\end{abstract}

Keywords: symmetric and quasisymmetric functions, omega operator, Schur functions

\section{Introduction}

Quasisymmetric functions have emerged as a powerful tool for investigating many diverse areas such as symmetric functions [2, 4], combinatorial Hopf algebras [1], discrete geometry [3], and representation theory [11, 13]. Quasisymmetric functions were introduced by Gessel as a source of generating functions for $P$-partitions [6], although they appeared in a different format in earlier work by Stanley [16]. Gessel developed many properties of quasisymmetric functions and applied them to solve a number of problems in permutation enumeration. Gessel also proved that they were dual to Solomon's descent algebra. This duality is further explored by Ehrenborg [5], Malvenuto and Reutenauer [14], and Thibon [19].

In [7], Haglund, Luoto, Mason, and van Willigenburg introduced a new basis for quasisymmetric functions called the quasisymmetric Schur function basis which are generated combinatorially through fillings of composition diagrams

\footnotetext{
${ }^{\dagger}$ Partially supported by NSF DMS Postdoc 0603351

$\ddagger$ Partially supported by NSF grant DMS 0654060 
in much the same way as Schur functions are generated through reverse column-strict tableaux. Each quasisymmetric Schur function is a positive sum of Demazure atoms. In [7], it was shown that the quasisymmetric Schur functions refine the Schur functions in a way that respects the Schur function decomposition into Gessel's fundamental quasisymmetric functions. In [8], Haglund, Luoto, Mason, and van Willigenburg gave a refinement of the LittlewoodRichardson rule which proved that the product of a quasisymmetric Schur function and a Schur function expands positively as a sum of quasisymmetric Schur functions.

This paper was motivated by an attempt to extend the duality between column-strict tableaux and row-strict tableaux to quasisymmetric Schur functions. That is, let $\lambda=\left(\lambda_{1}, \lambda_{2}, \ldots, \lambda_{k}\right)$ be a partition of $n$. The diagram associated to $\lambda$ (in English notation) consists of $k$ rows of left-justified boxes, or cells, such that the $i^{\text {th }}$ row from the top contains $\lambda_{i}$ cells. A reverse column-strict tableau T of shape $\lambda$ is a filling of the cells of $\lambda$ with positive integers so that the rows are weakly decreasing and the columns are strictly decreasing. A reverse row-strict tableau $T$ of shape $\lambda$ is a filling of the cells of $\lambda$ with positive integers so that the rows are strictly decreasing and the columns are weakly decreasing. Let $\mathcal{R} \mathcal{C} \mathcal{S}_{\lambda}\left(\mathcal{R} \mathcal{R} \mathcal{S}_{\lambda}\right)$ denote the set of all reverse column strict tableaux (reverse row strict tableaux) of shape $\lambda$. If $T$ is a reverse column-strict tableau or a reverse row-strict tableau, we let $T(i, j)$ be the element in the cell which is in the $i$-th row of $T$, reading from top to bottom, and the $j$-th column of $T$, reading from left to right, and we let the weight, $x^{T}$, of $T$ be defined as $x^{T}=\prod_{(i, j) \in \lambda} x_{T(i, j)}$. Then the Schur function $s_{\lambda}\left(x_{1}, x_{2}, \ldots\right)$ is defined as

$$
s_{\lambda}\left(x_{1}, x_{2}, \ldots\right)=\sum_{T \in \mathcal{R C} \mathcal{S}_{\lambda}} x^{T}
$$

If $T$ is a reverse column-strict tableau or a reverse row-strict tableau of shape $\lambda$, we define the conjugate of $T, T^{\prime}$, to be the filled diagram of shape $\lambda^{\prime}$ which results by reflecting the cells of $T$ across the main diagonal. Clearly $T$ is a reverse column-strict tableaux if and only if $T^{\prime}$ is a reverse-row strict tableaux. Thus

$$
s_{\lambda^{\prime}}\left(x_{1}, x_{2}, \ldots\right)=\sum_{T \in \mathcal{R} \mathcal{R} \mathcal{S}_{\lambda}} x^{T}
$$

where $\lambda^{\prime}$ is the transpose of the partition $\lambda$, often referred to as conjugate partition [9, 10, 18]. Moreover, if $\omega$ is the algebra isomorphism defined on the ring of symmetric functions $\Lambda$ so that $\omega\left(h_{n}\right)=e_{n}$, where $h_{n}=h_{n}\left(x_{1}, x_{2}, \ldots\right)$ is $n$-th homogeneous symmetric function and $e_{n}=e_{n}\left(x_{1}, x_{2}, \ldots\right)$ is $n$-th elementary symmetric function, then it is well known that $\omega\left(s_{\lambda}\left(x_{1}, x_{2}, \ldots\right)\right)=s_{\lambda^{\prime}}\left(x_{1}, x_{2}, \ldots\right)$.

The question that motivated this paper is whether we can find a duality like that expressed in (1) and (2) for quasisymmetric Schur functions. In this paper, we introduce a new basis for the quasisymmetric functions which we call row-strict quasisymmetric Schur functions which are generated combinatorially through fillings of composition diagrams in much the same way as Schur functions are generated through reverse row-strict tableaux. However, the process of conjugation becomes less transparent in the quasisymmetric setting since bases for quasisymmetric functions are typically indexed by compositions instead of partitions. That is, it is not enough to simply reflect across the main diagonal since this does not necesarily produce a left-justified diagram. In fact, the number of compositions which are rearrangements of a given partition is generally not equal to the number of compositions which are rearrangements of its transpose so that any relationship between these two collections must necessarily be more complex than a simple bijection. There is a refinement of the $\omega$ transformation which is defined on the space of quasisymmetric functions and we shall use this refinement to better understand the relationship between the compositions rearranging a partition and those rearranging its conjugate. 


\section{Symmetric and quasisymmetric functions}

A symmetric function is a bounded degree formal power series $f(x) \in \mathbb{Q}\left[\left[x_{1}, x_{2}, \ldots\right]\right]$ such that $f(x)$ is fixed under the action of the symmetric group. We let $\Lambda$ denote the ring of symmetric functions and $\Lambda_{n}$ denote the space of homogeneous symmetric functions of degree $n$ so that $\Lambda=\oplus_{n \geq 0} \Lambda_{n}$.

A partition of $n$ is a weakly decreasing sequence of positive integers which sum to $n$. We write $|\lambda|=n$ and let $l(n)=k$ be the length of $\lambda$. Given a partition $\lambda=\left(\lambda_{1}, \ldots, \lambda_{k}\right)$ of $n$, we say that a reverse column-strict tableau $T$ is a standard reverse column-strict tableau if each of the numbers $1,2, \ldots, n$ appear exactly once in $T$. Standard reverse row-strict tableaux are defined similarly. A reverse column-strict tableau can be converted to a standard reverse column-strict tableau by a procedure known as standardization. Let $T$ be an arbitrary reverse column-strict tableau such that $x^{T}=x_{1}^{a_{1}} x_{2}^{a_{2}} \ldots x_{k}^{a_{k}}$. First replace (from right to left) the $a_{1} 1 \mathrm{~s}$ in $T$ with the numbers $1,2, \ldots, a_{1}$. Then replace the $a_{2} 2 \mathrm{~s}$ with the numbers $a_{1}+1, \ldots, a_{1}+a_{2}$, and so on. The resulting diagram is a standard reverse columnstrict tableau, called the standardization $\operatorname{std}(T)$ of $T$. The standardization of a reverse row-strict tableau is defined analogously to that of a reverse column-strict tableau but with the entries replaced from bottom to top rather than right to left.

A composition $\alpha \vDash n$ of $n$ is a sequence of positive integers which sum to $n$. Each composition $\alpha=$ $\left(\alpha_{1}, \alpha_{2}, \ldots, \alpha_{k}\right)$ is associated to the subset of $[n-1]$ given by $S(\alpha)=\left\{\alpha_{1}, \alpha_{1}+\alpha_{2}, \ldots, \alpha_{1}+\alpha_{2}+\ldots \alpha_{k-1}\right\}$. Note that this is an invertible procedure. That is, if $P=\left\{s_{1}, s_{2}, \ldots, s_{k}\right\}$ is an arbitrary subset of $[n-1]$, then the composition $\boldsymbol{\beta}(P)=\left(s_{1}, s_{2}-s_{1}, \ldots, s_{k}-s_{k-1}\right)$ is precisely the composition such that $S(\boldsymbol{\beta}(P))=P$. We deonte by $\tilde{\alpha}$ the complement of $\alpha$, obtained by taking the composition corresponding to the complement of $S(\alpha)$. We will make use of the refinement order $\preceq$ on compositions which states that $\alpha \preceq \beta$ if and only if $\beta$ is obtained from $\alpha$ by summing some of the consecutive parts of $\alpha$. If $\alpha$ is a composition, let $\boldsymbol{\lambda}(\alpha)$ denote the partition obtained by arranging the parts of $\alpha$ in weakly decreasing order. We say that $\alpha$ is a rearrangement of the partition $\boldsymbol{\lambda}(\alpha)$. For example, $\boldsymbol{\lambda}(1,3,2,1)=(3,2,1,1)$.

A quasisymmetric function is a bounded degree formal power series $f(x) \in \mathbb{Q}\left[\left[x_{1}, x_{2}, \ldots\right]\right]$ such that for all compositions $\alpha=\left(\alpha_{1}, \alpha_{2}, \ldots, \alpha_{k}\right)$, the coefficient of $\prod x_{i}^{\alpha_{i}}$ is equal to the coefficient of $\prod x_{i_{j}}^{\alpha_{i}}$ for all $i_{1}<i_{2}<\ldots<i_{k}$. We let $Q$ sym denote the ring of quasisymmetric functions and $Q s y m_{n}$ denote the space of homogeneous quasisymmetric functions of degree $n$ so that $Q s y m=\oplus_{n \geq 0} Q s y m_{n}$.

A natural basis for sym $_{n}$ is the monomial quasisymmetric basis, given by the set of all $M_{\alpha}$ such that $\alpha \vDash n$ where

$$
M_{\alpha}=\sum_{i_{1}<i_{2}<\cdots<i_{k}} x_{i_{1}}^{\alpha_{1}} x_{i_{2}}^{\alpha_{2}} \cdots x_{i_{k}}^{\alpha_{k}} .
$$

Gessel's fundamental basis for quasisymmetric functions [6] can be expressed by

$$
F_{\alpha}=\sum_{\beta \preceq \alpha} M_{\beta},
$$

where $\beta \preceq \alpha$ means that $\beta$ is a refinement of $\alpha$.

The descent set $D(T)$ of a standard tableau is the set of all positive integers $i$ such that $i+1$ appears in a column weakly to the right of the column containing $i$. The following theorem provides describes the way a Schur function can be expressed as a positive sum of fundamental quasisymmetric functions.

Theorem 2.1 [6] The Schur function $s_{\lambda}$ can be written as a positive sum

$$
s_{\lambda}=\sum_{\beta} d_{\lambda \beta} F_{\beta}
$$


of fundamental quasisymmetric functions, where $d_{\lambda \beta}$ is equal to the number of standard reverse column-strict tableaux $T$ of shape $\lambda$ and descent set $D(T)$ such that $\boldsymbol{\beta}(D(T))=\beta$.

An extension of the classical $\omega$ transformation on symmetric functions defined in the introduction to the space of quasisymmetric functions appears in the work of Ehrenborg [5], Gessel [6], and Malvenuto-Reutenauer [14]. One can define this endomorphism on the fundamental quasisymmetric functions by $\omega\left(F_{\alpha}\right)=F_{\operatorname{rev}(\tilde{\alpha})}$, where $\operatorname{rev}(\tilde{\alpha})$ is the composition obtained by reversing the order of the entries in $\tilde{\alpha}$. Then $\omega$ is an automorphism of the algebra of quasisymmetric functions whose restriction to the space of symmetric function equals the classical $\omega$ transformation.

\section{Quasisymmetric Schur functions and row-strict quasisymmetric Schur functions}

Let $\alpha=\left(\alpha_{1}, \alpha_{2}, \ldots, \alpha_{l}\right)$ be a composition of $n$. The diagram associated to $\alpha$ consists of $l$ rows of left-justified boxes, or cells, such that the $i^{\text {th }}$ row from the top contains $\alpha_{i}$ cells, as in the English notation. Given a composition diagram $\alpha=\left(\alpha_{1}, \alpha_{2}, \ldots, \alpha_{l}\right)$ with largest part $m$, a column-strict composition tableau (CSCT), $F$ is a filling of the cells of $\alpha$ with positive integers such that the entries of $F$ weakly decrease in each row when read from left to right, the entries in the leftmost column of $F$ strictly increase when read from top to bottom, and $F$ satisfies the column-strict triple rule.

Here we say that $F$ satisfies the column-strict triple rule if when we supplement $F$ by adding enough cells with zero valued entries to the end of each row so that the resulting supplemented tableau, $\hat{F}$, is of rectangular shape $l \times m$, then for $1 \leq i<j \leq l, 2 \leq k \leq m$ :

$$
(\hat{F}(j, k) \neq 0 \text { and } \hat{F}(j, k) \geq \hat{F}(i, k)) \Rightarrow \hat{F}(j, k)>\hat{F}(i, k-1)
$$

where $\hat{F}(i, j)$ denotes the entry of $\hat{F}$ that lies in the cell in the $i$-th row and $j$-th column.

Define the type of a column-strict composition tableau $F$ to be the weak composition $w(F)=\left(w_{1}(F), w_{2}(F), \ldots\right)$ where $w_{i}(F)=$ the number of times $i$ appears in $F$. The weight of $F$ is $x^{F}=\prod_{i} x_{i}^{w_{i}(F)}$. A CSCT $F$ with $n$ cells is standard if $x^{F}=\prod_{i=1}^{n} x_{i}$. If $T$ is a standard CSCT, then we define the descent set $D(T)$ of $T$ to be the set of all $i$ such that $i+1$ appears in a column weakly to the right of the column containing $i$.

Haglund, Luoto, Mason, and van Willigenburg [7] defined the quasisymmetric Schur function $\mathcal{S}_{\alpha}$ by

$$
\mathcal{S}_{\alpha}=\sum_{F} x^{F}
$$

where the sum runs over all column-strict composition tableaux of shape $\alpha$. They showed that $\mathcal{S}_{\alpha}$ as $\alpha$ ranges over all compositions of $n$ is a basis for the space $Q s y m_{n}$. They also showed that for any partition $\lambda$ of $n$,

$$
s_{\lambda}=\sum_{\alpha: \lambda(\alpha)=\lambda} \mathcal{S}_{\alpha}
$$

and gave a combinatorial description of the expansion of $\mathcal{S}_{\alpha}$ in terms of the fundamental quasisymmetric function basis in the following proposition.

Proposition 3.1 [7. Proposition 6.2] Let $\alpha, \beta$ be compositions. Then

$$
\mathcal{S}_{\alpha}=\sum_{\beta} d_{\alpha \beta} F_{\beta},
$$

where $d_{\alpha \beta}=$ the number of standard column-strict composition tableaux $T$ of shape $\alpha$ and $\boldsymbol{\beta}(D(T))=\beta$. 


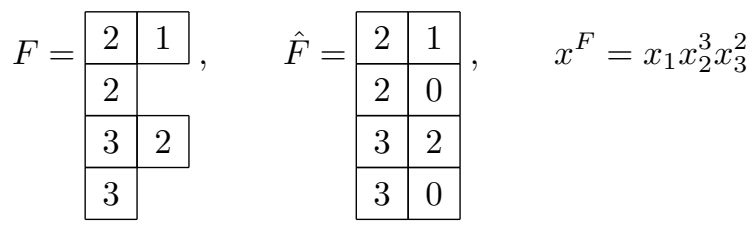

Fig. 1: An RSCT $F$ of shape $(2,1,2,1)$ and weight $x_{1} x_{2}^{3} x_{3}^{2}$

\begin{tabular}{|l|l|}
\hline 2 & 1 \\
\hline 2 & \\
\hline 3 & 2 \\
\hline 3 & \multicolumn{1}{|}{} \\
\cline { 1 - 1 }
\end{tabular}

\begin{tabular}{|l|l|}
\hline 2 & 1 \\
\hline 2 & \\
\hline 3 & 2 \\
\hline 4 & \multicolumn{1}{|l}{} \\
\cline { 1 - 2 }
\end{tabular}
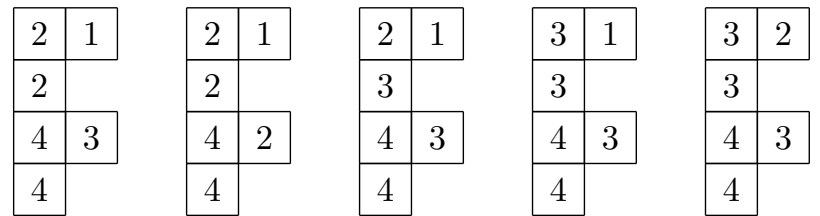

Fig. 2: The row-strict quasisymmetric Schur function $\mathcal{R S}_{(2,1,2,1)}\left(x_{1}, x_{2}, x_{3}, x_{4}\right)=x_{1} x_{2}^{3} x_{3}^{2}+x_{1} x_{2}^{3} x_{3} x_{4}+x_{1} x_{2}^{2} x_{3} x_{4}^{2}+$ $x_{1} x_{2}^{3} x_{4}^{2}+x_{1} x_{2} x_{3}^{2} x_{4}^{2}+x_{1} x_{3}^{3} x_{4}^{2}+x_{2} x_{3}^{3} x_{4}^{2}$

Next we define our row-strict version of the quasisymmetric Schur functions. Let $\alpha=\left(\alpha_{1}, \alpha_{2}, \ldots, \alpha_{l}\right)$ be a composition of $n$. Given a composition diagram $\alpha$ with largest part $m$, we define a row-strict composition tableau (RSCT), $F$ to be a filling of the cells of $\alpha$ with positive integers such that the entries of $F$ strictly decrease in each row when read from left to right, the entries in the leftmost column of $F$ weakly increase when read from top to bottom, and $F$ satisfies the row-strict triple rule.

Here we say that $F$ satisfies the row-strict triple rule if when we supplement $F$ by adding enough cells with zero valued entries to the end of each row so that the resulting supplemented tableau, $\hat{F}$, is of rectangular shape $l \times m$, then for $1 \leq i<j \leq l, 2 \leq k \leq m$ :

$$
(\hat{F}(j, k)>\hat{F}(i, k)) \Rightarrow \hat{F}(j, k) \geq \hat{F}(i, k-1) .
$$

This mirrors the definition of a composition tableau given in [7] and presented above, interchanging the roles of weak and strict. Continuing this analogy, define the type of a row-strict composition tableau $F$ to be the weak composition $w(F)=\left(w_{1}(F), w_{2}(F), \ldots\right)$ where $w_{i}(F)=$ the number of times $i$ appears in $F$. The weight associated to $F$ is $x^{F}=\prod_{i} x_{i}^{w_{i}(F)}$. Also, a RSCT $F$ with $n$ cells is standard if $x^{F}=\prod_{i=1}^{n} x_{i}$. See Figure 1 for an example of a RSCT and its weight.

Definition 3.1 Let $\alpha$ be a composition. Then the row-strict quasisymmetric Schur function $\mathcal{R} \mathcal{S}_{\alpha}$ is given by $\mathcal{R S}_{\alpha}=\sum_{T} x^{T}$, where the sum is over all RSCT's T of shape $\alpha$. See Figure 2 for an example.

Proposition 3.2 If $\alpha$ is an arbitrary composition, then $\mathcal{R} \mathcal{S}_{\alpha}$ is a quasisymmetric polynomial.

We shall see in Section 4.2 that the row-strict quasisymmetric Schur functions form a basis for quasisymmetric functions. The row-strict quasisymmetric Schur functions are a different basis from the quasisymmetric Schur functions even though they can be described through a similar process. For example, the transition matrix between the two bases is given in Figure 3 for $n=4$ where each row gives the expansion of $\mathcal{R} \mathcal{S}_{\alpha}$ in terms of the quasisymmetric Schur functions. This illustrates that their relationship is fairly complex. 


\begin{tabular}{l||c|c|c|c|c|c|c|c|}
$\mathcal{R} \mathcal{S}_{\alpha} \backslash \mathcal{S}_{\alpha}$ & 4 & 31 & 13 & 22 & 211 & 121 & 112 & 1111 \\
\hline \hline 4 & 0 & 0 & 0 & 0 & 0 & 0 & 0 & 1 \\
\hline 31 & 0 & 0 & 0 & 0 & 0 & 0 & 1 & 0 \\
\hline 13 & 0 & 0 & 0 & 0 & 1 & 1 & 0 & 0 \\
\hline 22 & 0 & 0 & 0 & 1 & 0 & 0 & 0 & 0 \\
\hline 211 & 0 & 0 & 1 & -1 & 0 & 1 & 0 & 0 \\
\hline 121 & 0 & 0 & 0 & 1 & 0 & -1 & 0 & 0 \\
\hline 112 & 0 & 1 & 0 & 0 & 0 & 0 & 0 & 0 \\
\hline 1111 & 1 & 0 & 0 & 0 & 0 & 0 & 0 & 0 \\
\hline
\end{tabular}

Fig. 3: The transition matrix from row-strict quasisymmetric Schur functions to quasisymmetric Schur functions

\subsection{Decomposing a Schur function into row-strict quasisymmetric Schur functions}

Recall that a reverse row-strict tableau $T$ of partition shape $\lambda$ is a filling of the cells of the diagram of $\lambda$ with positive integers such that the entries in each column weakly decrease from top to bottom and the entries in each row strictly decrease from left to right. Row-strict composition tableaux are related to reverse row-strict tableaux by a simple bijection, which is analogous to the bijection between column-strict composition tableaux and reverse column-strict tableaux [15].

The following map sends a reverse row-strict tableau $T$ to a RSCT $\rho(T)=F$. We describe it algorithmically. Begin with the entries in the leftmost column of $T$ and place them into the first column of $F$ in weakly increasing order from top to bottom. After the first $k-1$ columns of $T$ have been placed into $F$, place the entries from the $k^{\text {th }}$ column of $T$ into $F$, beginning with the largest. Place each entry $e$ into the cell $(i, k)$ in the highest row $i$ such that $(i, k)$ does not already contain an entry from $T$ and the entry $(i, k-1)$ is greater than $e$. See Figure 4 for an example of the map $\rho$ from a reverse row-strict tableau $T$ to a $\operatorname{RSCT} \rho(T)=F$.

Lemma 3.2 The map $\rho$ is a weight preserving bijection between the set of reverse row-strict tableaux of shape $\lambda$ and the set of RSCT's of shape $\alpha$ where $\lambda(\alpha)=\lambda$.

Lemma 3.2 implies that each Schur function $s_{\lambda}$ decomposes into a positive sum of row-strict quasisymmetric Schur functions indexed by compositions that rearrange the transpose of $\lambda$. That is,

$$
s_{\lambda}=\sum_{\alpha: \lambda(\alpha)=\lambda^{\prime}} \mathcal{R} \mathcal{S}_{\alpha}
$$

An arbitrary Schur function can therefore be decomposed into either a sum of quasisymmetric Schur functions [7] or a sum of row-strict quasisymmetric Schur functions.

\section{Properties of the row-strict quasisymmetric Schur functions}

In order to develop several fundamental properties of the row-strict quasisymmetric Schur functions, we need to understand the behavior of the map $\rho$ which interpolates between row-strict composition tableaux and reverse rowstrict tableaux. 


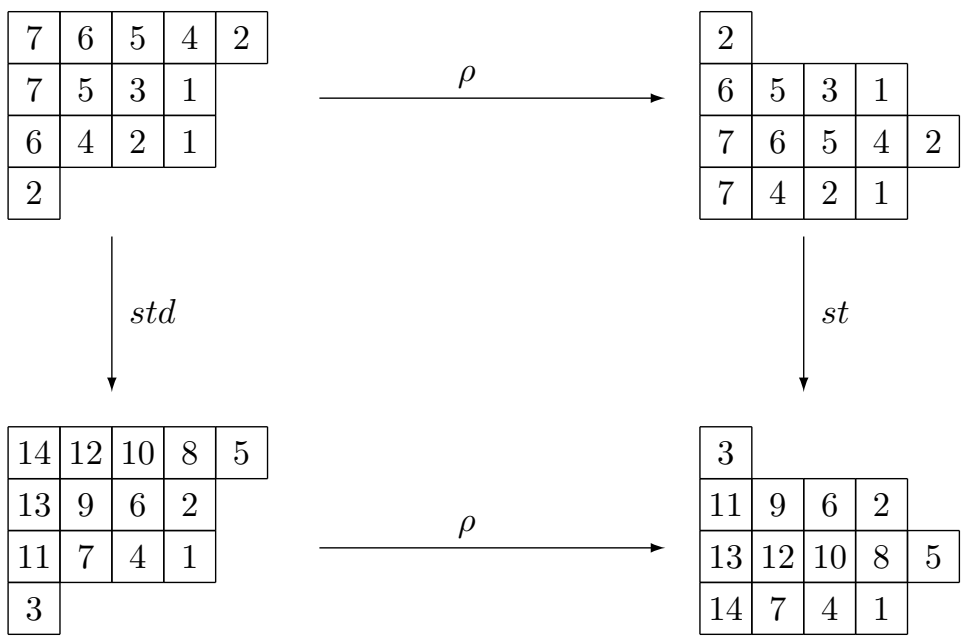

Fig. 4: The map $\rho$ commutes with standardization.

\subsection{Properties of the map $\rho$}

Recall that a reverse row-strict tableau can be converted to a standard tableau by the standardization procedure described in Section 2 The standardization of a row-strict composition tableau $F$ is defined similarly to that of a reverse row-strict tableau, where the entries are replaced beginning with the leftmost column and moving left to right, replacing entries within a column from bottom to top except for the entries in the leftmost column, which are replaced from top to bottom. The resulting standard composition tableau is called the standardization of $F$ and denoted $s t(F)$. See Figure 4 for an example. Note that this procedure does not alter the shape of $F$.

Proposition 4.1 Standardization commutes with the map $\rho$ in the sense that if $T$ is an arbitrary reverse row-strict tableau then $\operatorname{st}(\rho(T))=(\rho(\operatorname{std}(T))$.

\subsection{Transitions to classical quasisymmetric function bases}

Each monomial in a row-strict quasisymmetric Schur function corresponds to a row-strict composition tableau whose weight corresponds to the non-zero exponents in the monomial. Consider the monomial $x_{1}^{\beta_{1}} x_{2}^{\beta_{2}} \cdots x_{k}^{\beta_{k}}$ with exponent composition $\beta=\left(\beta_{1}, \beta_{2}, \ldots, \beta_{k}\right)$. The coefficient of this monomial in $\mathcal{R} \mathcal{S}_{\alpha}$ is equal to the number of row-strict composition tableaux of shape $\alpha$ and weight $\beta$. By shifting the entries in a row-strict composition tableau appropriately, it is easy to see that

$$
\mathcal{R S}_{\alpha}=\sum_{\beta} K(\alpha, \beta)^{r} M_{\beta}
$$

where $K(\alpha, \beta)^{r}$ is the number of row-strict composition tableaux of shape $\alpha$ and weight $\beta$.

Given a standard row-strict composition tableau $F$, its transpose descent set $D^{\prime}(F)$ is the set of all $i$ such that $i+1$ appears in a column strictly to the left of the column containing $i$. Since the map $\rho$ preserves the column sets of the diagram, the transpose descent set of a reverse row-strict standard Young tableau $T$ (defined analogously) is 
equal to the transpose descent set of $\rho(T)$. To each transpose descent set $S=\left\{s_{1}, s_{2}, \ldots s_{k}\right\}$ one associates a unique composition $\boldsymbol{\beta}(S)=\left(s_{1}, s_{2}-s_{1}, \ldots, s_{k}-s_{k-1}, n-s_{k}\right)$ whose successive parts are equal to the differences between consecutive elements of the set $S$ and whose last part is given by the difference of $n$ and the last element $s_{k}$ in the set $S$.

We also recall the notion of complementary compositions. The complement $\tilde{\beta}$ to a composition $\boldsymbol{\beta}(S)$ arising from a subset $S \subseteq[n-1]$ is the composition obtained from the subset $S^{c} \subseteq[n-1]$. For example, the composition $\beta=(1,4,2)$ arising from the subset $S=\{1,5\} \subseteq[7]$ has complement $\tilde{\beta}=(2,1,1,2,1)$ arising from the subset $S^{c}=\{2,3,4,6,7\}$.

Next we provide the analogue of Proposition 3.1 for row-strict quasisymmetric Schur functions.

Proposition 4.2 Let $\alpha, \beta$ be compositions. Then

$$
\mathcal{R S}_{\alpha}=\sum_{\beta} d_{\alpha \beta} F_{\beta}
$$

where $d_{\alpha \beta}$ is equal to the number of standard row-strict composition tableaux $F$ of shape $\alpha$ and $\beta\left(D^{\prime}(F)\right)=\beta$.

One can use Proposition 4.2 to show that under a proper ordering of the compositions of $n$, the transition matrix between the basis of row-strict quasisymmetric Schur functions of degree $n$ and the basis of fundamental quasisymmetric functions of degree $n$ is an upper triangular matrix with 1's on the diagonal. This implies the following theorem.

Theorem 4.1 The set $\left\{\mathcal{R S}_{\alpha}\left(\mathbf{x}_{\mathbf{k}}\right) \mid \alpha \vDash n\right.$ and $\left.k \geq n\right\}$ forms a $\mathbb{Z}$-basis for QSym ${ }_{n}\left(\mathbf{x}_{\mathbf{k}}\right)$, where $\mathbf{x}_{\mathbf{k}}=x_{1}, x_{2}, \ldots, x_{k}$.

\section{A linear endomorphism of QSym}

An algebra endomorphism $\omega: \Lambda \rightarrow \Lambda$ on symmetric functions is defined by $\omega\left(e_{n}\right)=h_{n}, n \geq 1$. The definitions of $e_{\lambda}$ and $h_{\lambda}$ imply that $\omega\left(e_{\lambda}\right)=h_{\lambda}$ since $\omega$ preserves multiplication. The endomorphism is an involution and $\omega\left(s_{\lambda}\right)=s_{\lambda^{\prime}}$, where $\lambda^{\prime}$ is the transpose of the partition $\lambda$, often referred to as conjugate partition [9, 10, 18].

Theorem 5.1 The $\omega$ operator maps quasisymmetric Schur functions to row-strict quasisymmetric Schur functions. That is, $\omega\left(\mathcal{S}_{\alpha}\left(x_{1}, \ldots, x_{n}\right)\right)=\mathcal{R S}_{\alpha}\left(x_{n}, \ldots, x_{1}\right)$.

To see this, apply $\omega$ to the decomposition of $\mathcal{S}_{\alpha}$ into a sum of fundamental quasisymmetric functions.

\subsection{A notion of conjugation for compositions}

The $\omega$ operator applied to a Schur function $s_{\lambda}$ produces the Schur function $s_{\lambda^{\prime}}$ indexed by the conjugate partition to $\lambda$. Since both the quasisymmetric Schur functions and the row-strict quasisymmetric Schur functions are generated by sums of monomials arising from fillings of composition diagrams, it is natural to seek a conjugation-like operation on composition diagrams.

This idea at first seems to be too much to ask since there cannot be a bijection between compositions that rearrange a given partition and compositions that rearrange its conjugate. Consider for example the partition $\lambda=(2,1,1)$. There are three compositions $((2,1,1),(1,2,1)$ and $(1,1,2))$ which rearrange $\lambda$ but only two compositions $((3,1)$ and $(1,3))$ which rearrange $\lambda^{\prime}=(3,1)$. However, the $\omega$ operator can be used to collect the compositions which rearrange a given partition so that there exists a bijection between these collections and the collections corresponding to the conjugate partition. In particular, note that since $\omega$ sends a quasisymmetric Schur function to a row-strict quasisymmetric Schur function, a method for writing the quasisymmetric Schur functions in terms of their duals under $\omega$ and vice versa 


$$
\begin{aligned}
& F=\begin{array}{|l|l|l|l|l|}
\hline 2 & 1 & 1 & \multicolumn{3}{|l}{} \\
\cline { 1 - 2 } 4 & \multicolumn{5}{|l}{} \\
\hline 5 & 5 & 5 & 3 & 1 \\
\hline 6 & 3 & 2 & 2 & \multicolumn{1}{|l}{}
\end{array} \\
& C_{1}=\{1,3,5,5,6\} \\
& C_{2}=\{2,2,3,5\} \\
& C_{3}=\{1,1,4\} \\
& C_{4}=\{2\}
\end{aligned}
$$

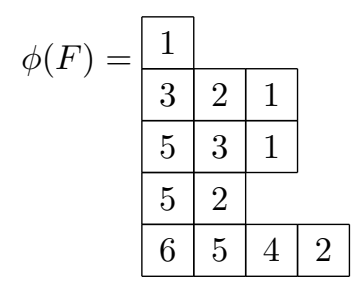

Fig. 5: The map $\phi$ from a $C S C T$ to an $R S C T$
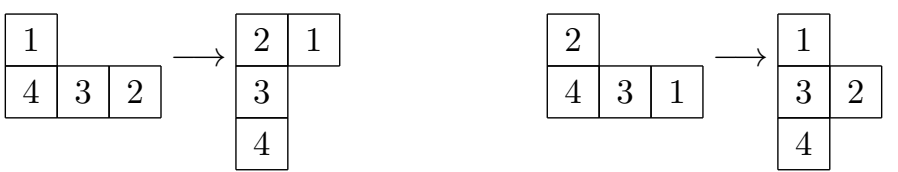

Fig. 6: The compositions $(2,1,1)$ and $(1,2,1)$ are conjugate to $(1,3)$

would allow us to interpret the indexing compositions in much the same way as we interpret the indexing partitions for Schur functions and their images under $\omega$.

Recall that the Schur functions can be expanded into sums of either quasisymmetric Schur functions or row-strict quasisymmetric Schur functions as follows:

$$
\sum_{\lambda(\alpha)=\lambda} \mathcal{S}_{\alpha}=s_{\lambda}=\sum_{\lambda(\beta)=\lambda^{\prime}} \mathcal{R S}_{\beta} .
$$

This suggests that there must be a weight-preserving bijection between column-strict composition tableaux and rowstrict composition tableaux which transposes the shape of the underlying partition.

Given an arbitrary column-strict composition tableau $F$, choose the largest entry in each column of $F$ and construct the leftmost column of $\phi(F)$ by placing these entries (the collection $C_{1}$ ) in weakly increasing order from top to bottom. Then choose the second-largest entry from each column of $F$ (ignoring empty cells) and insert this collection $\left(C_{2}\right)$ into the new diagram by the following procedure. Place the largest entry $e_{i}$ into the highest position in the second column so that the entry immediately to the left of $e_{i}$ is strictly greater than $e_{i}$. Repeat this insertion with the next largest entry, considering only the unoccupied positions. Continue this procedure with the remainder of the entries in this collection from largest to smallest until the entire collection has been inserted. Then repeat the procedure for the third largest entry in each column $\left(C_{3}\right)$ of $F$ and continue inserting collections of entries until all entries of $F$ have been inserted. The resulting diagram is $\phi(F)$. See Figure 5 for an example.

Proposition 5.1 The map $\phi$ is a weight-preserving bijection between column-strict composition tableaux which rearrange a given partition and row-strict composition tableaux which rearrange the conjugate partition.

\section{An extension of dual Schensted insertion}

Schensted insertion provides a method for inserting an arbitrary positive integer into an arbitrary column-strict tableau. This insertion process forms the foundation for the well-known Robinson-Schensted-Knuth (RSK) algorithm 

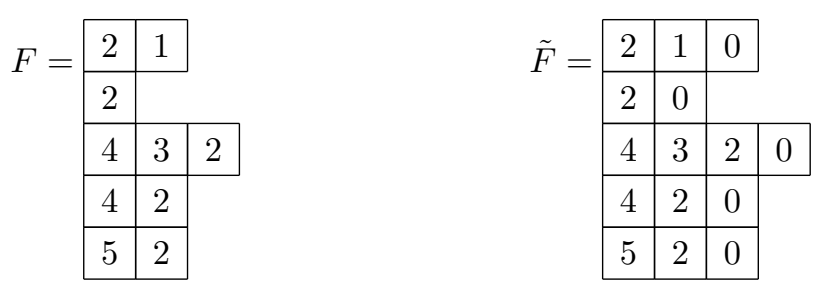

Fig. 7: $\operatorname{read}(\tilde{F})=002001032222445$

which produces a bijection between matrices with non-negative integer coefficients and pairs of reverse column-strict tableaux of the same shape. Mason [15] gave an extension of RSK algorithm to insert an integer into a column-strict composition tableaux and Haglund, Luoto, Mason and van Willigenburg [8] used this insertion procedure to prove the refinement of the Littlewood-Richardson rule which allows one to give a combinatorial interpretation to the coefficients that arise in the expansion the product of a Schur function times a quasisymmetric Schur function into a positive sum of quasisymmetric Schur functions.

In this section, we shall define an extension of dual Schensted insertion. This extension is used by Jeff Ferreira to give another refinement of the Littlewood-Richard rule which allows one to give a combinatorial interpretation to the coefficients that arise in the expansion the product of a Schur function times a row-strict quasisymmetric Schur functions into a positive sum of row-strict quasisymmetric Schur functions. We now describe our extension of the dual Schensted insertion to row-strict composition tableaux.

Dual Schensted insertion inserts an arbitrary positive integer into a reverse row-strict tableau by bumping entries from row to row. That is, given a reverse row-strict tableau $T$ and a positive integer $x$, first set $R$ equal to the first row of $T$. Let $y$ be the largest entry in $R$ less than or equal to $x$. Replace $y$ by $x$ in $R$. Set $x=y$ and set $R$ equal to the next row down and repeat. If there is no such entry $y$ which is less than or equal to $x$, place $x$ at the end of row $R$ and stop. The resulting figure is denoted $T \leftarrow x$. A more detailed exposition of Schensted insertion and its variations can be found in [17] or [12].

The following analogue of dual Schensted insertion provides a method for inserting a new cell into a RSCT. Given an arbitrary RSCT $F$, let read $(F)$ be the reading word for $F$ given by reading the entries of $F$ by column from right to left, reading the columns from top to bottom. This ordering of the cells is called the reading order on the cells of $F$. The modified reading word $\operatorname{read}(\tilde{F})$ for $F$ is given by appending a cell containing the entry 0 after the rightmost cell in each row to obtain $\tilde{F}$ and then recording the entries of $\tilde{F}$ in reading order. (See Figure 6 for an example.)

To insert an arbitrary positive integer $x$ into $F$, scan $\operatorname{read}(\tilde{F})$ to find the first entry $y$ less than or equal to $x$ such that the entry immediately to the left of $y$ in $\tilde{F}$ is greater than $x$. If such a $y$ does not exist place $x$ after the last entry smaller than or equal to $x$ in the leftmost column, shifting the lower rows down by one, and stop. If $y=0$ then replace $y$ by $x$ and stop. Otherwise, replace $y$ by $x$ in which case we say $x$ bumps $y$ and repeat the procedure using $y$ instead of $x$ and considering only the portion of $\operatorname{read}(\tilde{F})$ appearing after $y$. The resulting figure is denoted $F-x$. See Figure 8 for an example. Note that $x$ followed by all the elements which were bumped in the insertion procedure read in reading order must form a weakly decreasing sequence.

Lemma 6.1 The insertion procedure $F-x$ produces an $R S C T$.

Our extension of the dual Schensted algorithm has a number of nice properties. For example, we can prove that the following theorem. 


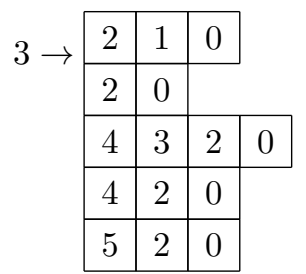

$3 \rightarrow$

002001032222445

\begin{tabular}{|l|l|l|l|}
\hline 2 & 1 & 0 & \multicolumn{2}{|c}{0} \\
\cline { 1 - 2 } 2 & 0 & \multicolumn{2}{|c}{} \\
\cline { 1 - 2 } 4 & $\mathbf{3}$ & 2 & 0 \\
\hline 4 & 2 & 0 & \multicolumn{1}{|c}{0} \\
\cline { 1 - 2 } 5 & 2 & 0 & \multicolumn{1}{|c}{} \\
\cline { 1 - 2 } & &
\end{tabular}

$3 \rightarrow \mathbf{2 2 2 2 4 4 5}$

\begin{tabular}{|l|l|l|l|}
\hline 2 & 1 & 0 & \multicolumn{2}{|c}{} \\
\cline { 1 - 2 } 2 & 0 & \multicolumn{2}{|c}{} \\
\hline 4 & 3 & 2 & 0 \\
\hline 4 & $\mathbf{3}$ & 0 & \multicolumn{1}{|}{} \\
\cline { 1 - 2 } 5 & 2 & 0 & \multicolumn{1}{|c}{} \\
\cline { 1 - 2 } & &
\end{tabular}

$2 \rightarrow 222445$

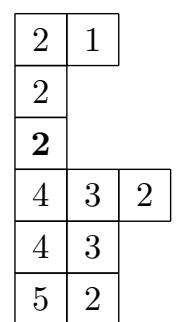

$F-3$

Fig. 8: The insertion procedure $F-3$

Theorem 6.2 The insertion procedure on RSCT commutes with the reverse row insertion in the sense that $\rho(T \leftarrow$ $x)=\rho(T)-x$.

\section{References}

[1] Marcelo Aguiar, Nantel Bergeron, and Frank Sottile. Combinatorial Hopf algebras and generalized DehnSommerville relations. Compos. Math., 142(1):1-30, 2006.

[2] Nantel Bergeron, Stefan Mykytiuk, Frank Sottile, and Stephanie van Willigenburg. Noncommutative Pieri operators on posets. J. Combin. Theory Ser. A, 91(1-2):84-110, 2000. In memory of Gian-Carlo Rota.

[3] Louis J. Billera, Samuel K. Hsiao, and Stephanie van Willigenburg. Peak quasisymmetric functions and Eulerian enumeration. Adv. Math., 176(2):248-276, 2003.

[4] Louis J. Billera, Hugh Thomas, and Stephanie van Willigenburg. Decomposable compositions, symmetric quasisymmetric functions and equality of ribbon Schur functions. Adv. Math., 204(1):204-240, 2006.

[5] R. Ehrenborg. On posets and Hopf algebras. advances in mathematics, 119(1):1-25, 1996.

[6] I.M. Gessel. Multipartite p-partitions and inner products of skew schur functions. Contemp. Math, 34:289-301, 1984.

[7] J. Haglund, K. Luoto, S. Mason, and S. van Willigenburg. Quasisymmetric Schur functions. Journal of Combinatorial Theory, Series A, 2009. to appear.

[8] J. Haglund, K. Luoto, S. Mason, and S. van Willigenburg. Refinements of the Littlewood-Richardson rule. Journal of Combinatorial Theory, Series A, 2009. to appear.

[9] P. Hall. The algebra of partitions. Proc. 4th Canadian Math. Congress (Banff), pages 147-159, 1959.

[10] P. Hall. The algebra of partitions. The collected works of Philip Hall, page 465, 1988.

[11] Florent Hivert. Hecke algebras, difference operators, and quasi-symmetric functions. Adv. Math., 155(2):181$238,2000$. 
[12] Donald E. Knuth. The art of computer programming. Volume 3. Addison-Wesley Publishing Co., Reading, Mass.-London-Don Mills, Ont., 1973. Sorting and searching, Addison-Wesley Series in Computer Science and Information Processing.

[13] Daniel Krob and Jean-Yves Thibon. Noncommutative symmetric functions. V. A degenerate version of $U_{q}\left(\mathrm{gl}_{N}\right)$. Internat. J. Algebra Comput., 9(3-4):405-430, 1999. Dedicated to the memory of Marcel-Paul Schützenberger.

[14] Clauda Malvenuto and Christophe Reutenauer. Duality between quasi-symmetric functions and the Solomon descent algebra. J. Algebra, 177(3):967-982, 1995.

[15] Sarah Mason. A decomposition of Schur functions and an analogue of the Robinson-Schensted-Knuth algorithm. Séminaire Lotharingien de Combinatoire, 57(B57e), 2008.

[16] Richard P. Stanley. Ordered structures and partitions. pages iii+104, 1972. Memoirs of the American Mathematical Society, No. 119.

[17] Richard P. Stanley. Enumerative combinatorics. Vol. 2, volume 62 of Cambridge Studies in Advanced Mathematics. Cambridge University Press, Cambridge, 1999. With a foreword by Gian-Carlo Rota and appendix 1 by Sergey Fomin.

[18] RP Stanley. Theory and applications of plane partitions: Part 1, 2. Stud. Appl. Math, 50:167-188, 1971.

[19] Jean-Yves Thibon. Lectures on noncommutative symmetric functions. 11:39-94, 2001. 